\title{
THE ESSENTIAL NORMS OF COMPOSITION OPERATORS BETWEEN GENERALIZED BLOCH SPACES IN THE POLYDISC AND THEIR APPLICATIONS
}

ZEHUA ZHOU AND YAN LIU

Received 27 December 2005; Revised 26 June 2006; Accepted 22 July 2006

Let $U^{n}$ be the unit polydisc of $\mathbb{C}^{n}$ and $\phi=\left(\phi_{1}, \ldots, \phi_{n}\right)$ a holomorphic self-map of $U^{n}$. $\mathscr{B}_{P}^{P}\left(U^{n}\right), \mathscr{B}_{0}^{P}\left(U^{n}\right)$, and $\mathscr{B}_{0_{*}}^{p}\left(U^{n}\right)$ denote the $p$-Bloch space, little $p$-Bloch space, and little star $p$-Bloch space in the unit polydisc $U^{n}$, respectively, where $p, q>0$. This paper gives the estimates of the essential norms of bounded composition operators $C_{\phi}$ induced by $\phi$ between $\mathscr{B}^{p}\left(U^{n}\right)\left(\mathscr{B}_{0}^{p}\left(U^{n}\right)\right.$ or $\left.\mathscr{B}_{0_{*}}^{p}\left(U^{n}\right)\right)$ and $\mathscr{B}^{q}\left(U^{n}\right)\left(\mathscr{B}_{0}^{q}\left(U^{n}\right)\right.$ or $\left.\mathscr{B}_{0_{*}}^{q}\left(U^{n}\right)\right)$. As their applications, some necessary and sufficient conditions for the (bounded) composition operators $C_{\phi}$ to be compact from $\mathscr{B}^{p}\left(U^{n}\right)\left(\mathscr{B}_{0}^{p}\left(U^{n}\right)\right.$ or $\left.\mathscr{B}_{0_{*}}^{p}\left(U^{n}\right)\right)$ into $\mathscr{B}^{q}\left(U^{n}\right)\left(\mathscr{B}_{0}^{q}\left(U^{n}\right)\right.$ or $\left.\mathscr{B}_{0_{*}}^{q}\left(U^{n}\right)\right)$ are obtained.

Copyright (c) 2006 Z. Zhou and Y. Liu. This is an open access article distributed under the Creative Commons Attribution License, which permits unrestricted use, distribution, and reproduction in any medium, provided the original work is properly cited.

\section{Introduction}

The class of all holomorphic functions with domain $\Omega$ will be denoted by $H(\Omega)$, where $\Omega$ is a bounded homogeneous domain in $\mathbb{C}^{n}$. Let $\phi$ be a holomorphic self-map of $\Omega$, the composition operator $C_{\phi}$ induced by $\phi$ is defined by

$$
\left(C_{\phi} f\right)(z)=f(\phi(z))
$$

for $z$ in $\Omega$ and $f \in H(\Omega)$.

Let $K(z, z)$ be the Bergman kernel function of $\Omega$, the Bergman metric $H_{z}(u, u)$ in $\Omega$ is defined by

$$
H_{z}(u, u)=\frac{1}{2} \sum_{j, k=1}^{n} \frac{\partial^{2} \log K(z, z)}{\partial z_{j} \partial \bar{z}_{k}} u_{j} \bar{u}_{k}
$$

where $z \in \Omega$ and $u=\left(u_{1}, \ldots, u_{n}\right) \in \mathbb{C}^{n}$. 
Following Timoney [5], we say that $f \in H(\Omega)$ is in the Bloch space $\mathscr{B}(\Omega)$ if

$$
\|f\|_{\mathscr{B}(\Omega)}=\sup _{z \in \Omega} Q_{f}(z)<\infty
$$

where

$$
Q_{f}(z)=\sup \left\{\frac{|\nabla f(z) u|}{H_{z}^{1 / 2}(u, u)}: u \in \mathbb{C}^{n}-\{0\}\right\},
$$

and $\nabla f(z)=\left(\partial f(z) / \partial z_{1}, \ldots, \partial f(z) / \partial z_{n}\right), \nabla f(z) u=\sum_{l=1}^{n}\left(\partial f(z) / \partial z_{l}\right) u_{l}$.

The little Bloch space $\mathscr{B}_{0}(\Omega)$ is the closure in the Banach space $\mathscr{B}(\Omega)$ of the polynomial functions.

Let $\partial \Omega$ denote the boundary of $\Omega$. Following Timoney [6], for $\Omega=B_{n}$ the unit ball of $\mathbb{C}^{n}, \mathscr{B}_{0}\left(B_{n}\right)=\left\{f \in \mathscr{B}_{(}\left(B_{n}\right): Q_{f}(z) \rightarrow 0\right.$, as $\left.z \rightarrow \partial B_{n}\right\}$; for $\Omega=\mathscr{D}$ the bounded symmetric domain other than the ball $B_{n},\left\{f \in \mathscr{B}(\mathscr{D}): Q_{f}(z) \rightarrow 0\right.$, as $\left.z \rightarrow \partial \mathscr{D}\right\}$ is the set of constant functions on $\mathscr{D}$. So if $\mathscr{D}$ is a bounded symmetric domain other than the ball, we denote the $\mathscr{B}_{0 *}(\mathscr{D})=\left\{f \in \mathscr{B}(\mathscr{D}): Q_{f}(z) \rightarrow 0\right.$, as $\left.z \rightarrow \partial^{*} \mathscr{D}\right\}$ and call it little star Bloch space; here $\partial^{*} \mathscr{D}$ means the distinguished boundary of $\mathscr{D}$. The unit ball is the only bounded symmetric domain $\mathscr{D}$ with the property that $\partial^{*} \mathscr{D}=\partial \mathscr{D}$.

Let $U^{n}$ be the unit polydisc of $\mathbb{C}^{n}$. Timoney [5] shows that $f \in \mathscr{B}\left(U^{n}\right)$ if and only if

$$
\|f\|_{1}=|f(0)|+\sup _{z \in U^{n}} \sum_{k=1}^{n}\left|\frac{\partial f}{\partial z_{k}}(z)\right|\left(1-\left|z_{k}\right|^{2}\right)<+\infty,
$$

where $f \in H\left(U^{n}\right)$.

This definition was the starting point for introducing the $p$-Bloch spaces.

Let $p>0$, a function $f \in H\left(U^{n}\right)$ is said to belong to the $p$-Bloch space $\mathscr{B}^{p}\left(U^{n}\right)$ if

$$
\|f\|_{p}=|f(0)|+\sup _{z \in U^{n}} \sum_{k=1}^{n}\left|\frac{\partial f}{\partial z_{k}}(z)\right|\left(1-\left|z_{k}\right|^{2}\right)^{p}<+\infty .
$$

It is an easy exercise to show that $\mathscr{B}^{p}\left(U^{n}\right)$ is a Banach space with the norm $\|\cdot\|_{p}$ for $p \geq 1$; and for $0<p<1, \mathscr{B}^{p}\left(U^{n}\right)$ is a nonlocally convex topological vector space and $d(f, g)=\|f-g\|_{p}^{p}$ is a complete metric for it. Its proof idea is basic, we refer the reader to see the proof of Proposition 3.1 or the statement corresponding the Bloch-type space for the unit ball in [13].

Just like Timoney [6], if

$$
\lim _{z \rightarrow \partial U^{n}} \sum_{k=1}^{n}\left|\frac{\partial f}{\partial z_{k}}(z)\right|\left(1-\left|z_{k}\right|^{2}\right)^{p}=0,
$$

it is easy to show that $f$ must be a constant. Indeed, for fixed $z_{1} \in U,\left(\partial f / \partial z_{1}\right)(z)(1-$ $\left.\left|z_{1}\right|^{2}\right)^{p}$ is a holomorphic function in $z^{\prime}=\left(z_{2}, \ldots, z_{n}\right) \in U^{n-1}$. If $z \rightarrow \partial U^{n}$, then $z^{\prime} \rightarrow \partial U^{n-1}$, which implies that

$$
\lim _{z^{\prime} \rightarrow \partial U^{n-1}}\left|\frac{\partial f}{\partial z_{1}}(z)\right|\left(1-\left|z_{1}\right|^{2}\right)^{p}=0 .
$$


Hence, $\left(\partial f / \partial z_{1}\right)(z)\left(1-\left|z_{1}\right|^{2}\right)^{p} \equiv 0$ for every $z^{\prime} \in \partial U^{n-1}$, and for each $z_{1} \in U$, and consequently $\left(\partial f / \partial z_{1}\right)(z)=0$ for every $z \in U^{n}$. Similarly, we can obtain that $\left(\partial f / \partial z_{j}\right)(z)=0$ for every $z_{j} \in U^{n}$ and each $j \in\{2, \ldots, n\}$; therefore $f \equiv$ const.

So, there is no sense to introduce the corresponding little $p$-Bloch space in this way. We will say that the little $p$-Bloch space $\mathscr{B}_{0}^{p}\left(U^{n}\right)$ is the closure of the polynomials in the $p$-Bloch space. If $f \in H\left(U^{n}\right)$ and

$$
\sup _{z \in \partial^{*} U^{n}} \sum_{k=1}^{n}\left|\frac{\partial f}{\partial z_{k}}(z)\right|\left(1-\left|z_{k}\right|^{2}\right)^{p}=0
$$

we say $f$ belongs to little star $p$-Bloch space $\mathscr{S}_{0 *}^{p}\left(U^{n}\right)$. Using the same methods as that of [6, Theorem 4.15], we can show that $\mathscr{B}_{0}^{p}\left(U^{n}\right)$ is a proper subspace of $\mathscr{B}_{0 *}^{p}\left(U^{n}\right)$ and $\mathscr{S}_{0 *}^{p}\left(U^{n}\right)$ is a nonseparable closed subspace of $\mathscr{B}_{P} P\left(U^{n}\right)$.

For the unit disc $U \subset \mathbb{C}$, Madigan and Matheson [1] proved that $C_{\phi}$ is always bounded on $\mathscr{B}(U)$ and bounded on $\mathscr{B}_{0}(U)$ if and only if $\phi \in \mathscr{B}_{0}(U)$. They also gave the sufficient and necessary conditions that $C_{\phi}$ is compact on $\mathscr{B}(U)$ or $\mathscr{B}_{0}(U)$.

The analogues of these facts for the unit polydisc and classical symmetric domains were obtained by Zhou and Shi in [8-10]. They had already shown that $C_{\phi}$ is always bounded on the Bloch space of these domains, and also gave some sufficient and necessary conditions for $C_{\phi}$ to be compact on those spaces. For the results on the unit ball, we refer the reader to see $[4,12]$.

We recall that the essential norm of a continuous linear operator $T$ is the distance from $T$ to the compact operators, that is,

$$
\|T\|_{e}=\inf \{\|T-K\|: K \text { is compact }\}
$$

Notice that $\|T\|_{e}=0$ if and only if $T$ is compact, so that estimates on $\|T\|_{e}$ lead to conditions for $T$ to be compact.

As we have known that $C_{\phi}$ is always bounded on the Bloch space in the unit disc and polydisc, in [2], Montes-Rodriguez gave the exact essential norm of a composition operator on the Bloch space in the disc and obtained a different proof for the corresponding compactness results in [1]. After that, Zhou and Shi generalized Alsonso's result to the polydisc in [11].

In [7], Zhou stated and proved the corresponding compactness characterization for $\mathscr{B} P\left(U^{n}\right)$ for $0<p<1$, however, $C_{\phi}$ is not always bounded, and the test functions used in [7] are only suitable for handling the case $0<p<1$. It is therefore natural to wonder what results can be proven about boundedness and compactness of $C_{\phi}$ on $p$-Bloch spaces for an arbitrary positive number $p$ or, more generally, between possibly different $p$ - and $q$-Bloch spaces of multivariable domains. In this paper, we answer these questions completely for $U^{n}$ with essential norm approach, we give some estimates of the essential norms of bounded composition operators $C_{\phi}$ between $\mathscr{B}^{p}\left(U^{n}\right)\left(\mathscr{R}_{0}^{p}\left(U^{n}\right)\right.$ or $\left.\mathscr{H}_{0 *}^{p}\left(U^{n}\right)\right)$ and $\mathscr{B}^{q}\left(U^{n}\right)\left(\mathscr{B}_{0}^{q}\left(U^{n}\right)\right.$ or $\left.\mathscr{S}_{0 *}^{q}\left(U^{n}\right)\right)$. Further, we apply these results to obtain some necessary and sufficient conditions for the composition operators $C_{\phi}$ to be compact from $\mathscr{B}^{p}\left(U^{n}\right)\left(\mathscr{B}_{0}^{p}\left(U^{n}\right)\right.$ or $\left.\mathscr{H}_{0 *}^{p}\left(U^{n}\right)\right)$ into $\mathscr{B}^{q}\left(U^{n}\right)\left(\mathscr{B}_{0}^{q}\left(U^{n}\right)\right.$ or $\left.\mathscr{B}_{0 *}^{q}\left(U^{n}\right)\right)$. The fundamental 
ideas of the proof are those used by Shapiro [3] to obtain the essential norm of a composition operator on Hilbert spaces of analytic functions (Hardy and weighted Bergman spaces) in terms of natural counting functions associated with $\phi$. This paper generalizes the results on the Bloch space for the unit disc in [2] and the unit polydisc in [11].

Throughout the remainder of this paper $C$ will denote a positive constant, the exact value of which will vary from one appearance to the next.

Our main results are the following.

Theorem 1.1. Let $\phi=\left(\phi_{1}, \phi_{2}, \ldots, \phi_{n}\right)$ be a holomorphic self-map of $U^{n}$ and $\left\|C_{\phi}\right\|_{e}$ the essential norm of a bounded composition operator $C_{\phi}: \mathscr{B}^{p}\left(U^{n}\right)\left(\mathscr{B}_{0}^{p}\left(U^{n}\right)\right.$ or $\left.\mathscr{B}_{0 *}^{p}\left(U^{n}\right)\right) \rightarrow$ $\mathscr{B}^{q}\left(U^{n}\right)\left(\mathscr{B}_{0}^{q}\left(U^{n}\right)\right.$ or $\left.\mathscr{S}_{0 *}^{q}\left(U^{n}\right)\right)$, then

$$
\begin{aligned}
& \frac{1}{n} \lim _{\delta \rightarrow 0} \sup _{\operatorname{dist}\left(\phi(z), \partial U^{n}\right)<\delta} \sum_{k, l=1}^{n}\left|\frac{\partial \phi_{l}}{\partial z_{k}}(z)\right| \frac{\left(1-\left|z_{k}\right|^{2}\right)^{q}}{\left(1-\left|\phi_{l}(z)\right|^{2}\right)^{p}} \\
& \quad \leq\left\|C_{\phi}\right\|_{e} \leq 2 \lim _{\delta \rightarrow 0} \sup _{\operatorname{dist}\left(\phi(z), \partial U^{n}\right)<\delta} \sum_{k, l=1}^{n}\left|\frac{\partial \phi_{l}}{\partial z_{k}}(z)\right| \frac{\left(1-\left|z_{k}\right|^{2}\right)^{q}}{\left(1-\left|\phi_{l}(z)\right|^{2}\right)^{p}} .
\end{aligned}
$$

By Theorem 1.1 and the fact that $C_{\phi}: \mathscr{B}^{p}\left(U^{n}\right)\left(\right.$ or $\mathscr{B}_{0}^{p}\left(U^{n}\right)$ or $\left.\mathscr{B}_{0 *}^{p}\left(U^{n}\right)\right) \rightarrow \mathscr{B}^{q}\left(U^{n}\right)$ (or $\mathscr{B}_{0}^{q}\left(U^{n}\right)$ or $\mathscr{S}_{0 *}^{q}\left(U^{n}\right)$ ) is compact if and only if $\left\|C_{\phi}\right\|_{e}=0$, we obtain Theorem 1.2 at once.

THeOREM 1.2. Let $\phi=\left(\phi_{1}, \ldots, \phi_{n}\right)$ be a holomorphic self-map of $U^{n}$. Then the bounded composition operator $C_{\phi}: \mathscr{B}^{p}\left(U^{n}\right)\left(\mathscr{B}_{0}^{p}\left(U^{n}\right)\right.$ or $\left.\mathscr{B}_{0 *}^{p}\left(U^{n}\right)\right) \rightarrow \mathscr{B}^{q}\left(U^{n}\right)\left(\mathscr{B}_{0}^{q}\left(U^{n}\right)\right.$ or $\left.\mathscr{B}_{0 *}^{q}\left(U^{n}\right)\right)$ is compact if and only if for any $\varepsilon>0$, there exists a $\delta$ with $0<\delta<1$, such that

$$
\sup _{\operatorname{dist}\left(\phi(z), \partial U^{n}\right)<\delta} \sum_{k, l=1}^{n}\left|\frac{\partial \phi_{l}}{\partial z_{k}}(z)\right| \frac{\left(1-\left|z_{k}\right|^{2}\right)^{q}}{\left(1-\left|\phi_{l}(z)\right|^{2}\right)^{p}}<\varepsilon .
$$

Remark 1.3. When $n=1, p=q=1$, on $\mathscr{B}(U)$ we obtain [1, Theorem 2]. Since $\partial U=$ $\partial^{*} U, \mathscr{B}_{0}(U)=\mathscr{B}_{0 *}(U)$, we can also obtain [1, Theorem 1$]$.

Remark 1.4. When $n>1, p=q=1, C_{\phi}$ is always bounded on $\mathscr{B}\left(U^{n}\right)$, so we can obtain the corresponding results in $[8,11]$.

The remainder of the present paper is assembled as follows: in Section 2, we state some lemmas for the proof of Theorem 1.1. In terms of mapping properties of symbol $\phi$, Lemmas $2.3,2.4$, and 2.6 will give some conditions for $C_{\phi}$ to be bounded between possibly different $p$ - and $q$-Bloch spaces, "little" or "little star" $p$ - and $q$-Bloch spaces, the methods used are different from that of [7], since the test functions used in [7] are only suitable for handling the $p$-Bloch space for the case $0<p<1$, not others. In Section 3, we give the proof of Theorem 1.1. In Section 4, as applications of Theorems 1.1 and 1.2, we give some corollaries for $C_{\phi}$ to be compact on those spaces. 


\section{Some lemmas}

In order to prove Theorem 1.1, we need some lemmas.

LEMma 2.1. Let $f \in \mathscr{B}_{P}\left(U^{n}\right)$, then

(1) if $0 \leq p<1$, then $|f(z)| \leq|f(0)|+(n /(1-p))\|f\|_{p}$;

(2) if $p=1$, then $|f(z)| \leq(1+1 / n \ln 2)\left(\sum_{k=1}^{n} \ln \left(2 /\left(1-\left|z_{k}\right|^{2}\right)\right)\right)\|f\|_{p}$;

(3) if $p>1$, then $|f(z)| \leq\left(1 / n+2^{p-1} /(p-1)\right) \sum_{k=1}^{n}\left(1 /\left(1-\left|z_{k}\right|^{2}\right)^{p-1}\right)\|f\|_{p}$.

Proof. This Lemma can be easily obtained by some integral estimates, so we omit the detail.

LeMma 2.2. For $p>0$, set

$$
f_{w}(z)=\int_{0}^{z_{l}} \frac{d t}{(1-\bar{w} t)^{p}}
$$

where $w \in U$. Then $f \in \mathscr{B}_{0}^{p}\left(U^{n}\right) \subset \mathscr{B}_{0 *}^{p}\left(U^{n}\right) \subset \mathscr{B}^{P}\left(U^{n}\right)$.

Proof. Since

$$
\frac{\partial f_{w}}{\partial z_{l}}=\left(1-\bar{w} z_{l}\right)^{-p}, \quad \frac{\partial f_{w}}{\partial z_{i}}=0, \quad i \neq l
$$

it follows that

$$
|f(0)|+\sum_{k=1}^{n}\left|\frac{\partial f_{w}}{\partial z_{k}}(z)\right|\left(1-\left|z_{k}\right|^{2}\right)^{p}=\frac{\left(1-\left|z_{l}\right|^{2}\right)^{p}}{\left|1-\bar{w} z_{l}\right|^{p}} \leq\left(1+\left|z_{l}\right|\right)^{p} \leq 2^{p}
$$

Hence $f_{w} \in \mathscr{B}^{p}\left(U^{n}\right)$.

Now we prove that $f_{w} \in \mathscr{B}_{0}^{p}\left(U^{n}\right)$. Using the asymptotic formula

$$
(1-\bar{w} t)^{-p}=\sum_{k=0}^{+\infty} \frac{p(p+1) \cdots(p+k-1)}{k !}(\bar{w})^{k} t^{k}
$$

we obtain

$$
f_{w}(z)=\sum_{k=0}^{+\infty} \frac{p(p+1) \cdots(p+k-1)}{k !}(\bar{w})^{k} \int_{0}^{z_{l}} t^{k} d t
$$

Denoting $P_{n}(z)=\sum_{k=0}^{n}(p(p+1) \cdots(p+k-1) / k !)(\bar{w})^{k} \int_{0}^{z_{l}} t^{k} d t$, it is easy to see that

$$
\left|\frac{\partial\left(f_{w}-P_{n}\right)}{\partial z_{l}}\right| \leq \sum_{k=n+1}^{+\infty} \frac{p(p+1) \cdots(p+k-1)}{k !}|w|^{k} \longrightarrow 0, \quad \text { as } n \longrightarrow \infty
$$


Thus

$$
\begin{aligned}
\left\|f_{w}-P_{n}\right\|_{p} & =\left|f_{w}(0)-P_{n}(0)\right|+\sup _{z \in U^{n}}\left|\frac{\partial\left(f_{w}-P_{n}\right)}{\partial z_{l}}\right|\left(1-\left|z_{l}\right|^{2}\right)^{p} \\
& \leq \sup _{z \in U^{n}}\left|\frac{\partial\left(f_{w}-P_{n}\right)}{\partial z_{l}}\right| \longrightarrow 0
\end{aligned}
$$

which shows that $f_{w} \in \mathscr{B}_{0}^{p}\left(U^{n}\right)$. So $f \in \mathscr{B}_{0}^{p}\left(U^{n}\right) \subset \mathscr{B}_{0 *}^{p}\left(U^{n}\right) \subset \mathscr{B}^{p}\left(U^{n}\right)$.

Lemma 2.3. Let $\phi=\left(\phi_{1}, \ldots, \phi_{n}\right)$ be a holomorphic self-map of $U^{n}, p, q>0$. Then $C_{\phi}$ : $\mathscr{S}^{p}\left(U^{n}\right)\left(\mathscr{B}_{0}^{p}\left(U^{n}\right)\right.$ or $\left.\mathscr{B}_{0 *}^{p}\left(U^{n}\right)\right) \rightarrow \mathscr{B}^{q}\left(U^{n}\right)$ is bounded if and only if there exists a constant $C$ such that

$$
\sum_{k, l=1}^{n}\left|\frac{\partial \phi_{l}}{\partial z_{k}}(z)\right| \frac{\left(1-\left|z_{k}\right|^{2}\right)^{q}}{\left(1-\left|\phi_{l}(z)\right|^{2}\right)^{p}} \leq C
$$

for all $z \in U^{n}$.

Proof. First assume that condition (2.8) holds and let $f \in \mathscr{B}^{p}\left(U^{n}\right)$. By Lemma 2.1, we know the evaluation at $\phi(0)$ is a bounded linear functional on $\mathscr{R}^{P}\left(U^{n}\right)$, so $|f(\phi(0))| \leq$ $C\|f\|_{p}$.

On the other hand we have

$$
\begin{aligned}
& \sum_{k=1}^{n}\left|\frac{\partial\left(C_{\phi} f(z)\right)}{\partial z_{k}}\right|\left(1-\left|z_{k}\right|^{2}\right)^{q}=\sum_{k=1}^{n}\left|\sum_{l=1}^{n} \frac{\partial f}{\partial \phi_{l}}(\phi(z)) \frac{\partial \phi_{l}}{\partial z_{k}}(z)\right|\left(1-\left|z_{k}\right|^{2}\right)^{q} \\
& \quad \leq \sum_{k, l=1}^{n}\left|\frac{\partial f}{\partial \phi_{l}}(\phi(z)) \frac{\partial \phi_{l}}{\partial z_{k}}(z)\right|\left(1-\left|z_{k}\right|^{2}\right)^{q} \\
& \quad \leq \sum_{l=1}^{n}\left|\frac{\partial f}{\partial \phi_{l}}(\phi(z))\right|\left(1-\left|\phi_{l}(z)\right|^{2}\right)^{p} \sum_{k, l=1}^{n}\left|\frac{\partial \phi_{l}}{\partial z_{k}}(z)\right| \frac{\left(1-\left|z_{k}\right|^{2}\right)^{q}}{\left(1-\left|\phi_{l}(z)\right|^{2}\right)^{p}} \\
& \quad \leq\|f\|_{p} \sum_{k, l=1}^{n}\left|\frac{\partial \phi_{l}}{\partial z_{k}}(z)\right| \frac{\left(1-\left|z_{k}\right|^{2}\right)^{q}}{\left(1-\left|\phi_{l}(z)\right|^{2}\right)^{p}} \leq C\|f\|_{p} .
\end{aligned}
$$

So $C_{\phi}: \mathscr{B}^{p}\left(U^{n}\right) \rightarrow \mathscr{B}^{q}\left(U^{n}\right)$ is bounded.

For the converse, assume that $C_{\phi}: \mathscr{B} p\left(U^{n}\right) \rightarrow \mathscr{B} q\left(U^{n}\right)$ is bounded, with

$$
\left\|C_{\phi} f\right\|_{q} \leq C\|f\|_{p}
$$

for all $f \in \mathscr{B}^{p}\left(U^{n}\right)$.

For fixed $l(1 \leq l \leq n)$, we will make use of a family of test functions $\left\{f_{w}: w \in \mathbb{C},|w|<\right.$ 1) defined in Lemma 2.2. 
Since

$$
f_{w} \in \mathscr{B}_{0}^{p}\left(U^{n}\right) \subset \mathscr{B}_{0 *}^{p}\left(U^{n}\right) \subset \mathscr{B}^{p}\left(U^{n}\right)
$$

it follows from (2.10) that for $z \in U^{n}$,

$$
\sum_{k=1}^{n}\left|\sum_{l=1}^{n} \frac{\partial f_{w}(\phi(z))}{\partial \phi_{l}} \frac{\partial \phi_{l}}{\partial z_{k}}(z)\right|\left(1-\left|z_{k}\right|^{2}\right)^{q} \leq C
$$

Let $w=\phi_{l}(z)$. Then

$$
\sum_{k=1}^{n}\left|\frac{\partial \phi_{l}}{\partial z_{k}}(z)\right| \frac{\left(1-\left|z_{k}\right|^{2}\right)^{q}}{\left(1-\left|\phi_{l}(z)\right|^{2}\right)^{p}} \leq C
$$

The results are stated above for $\mathscr{B}^{P}\left(U^{n}\right)$, but they also hold with minor modifications for $\mathscr{B}_{0}^{p}\left(U^{n}\right)$ and $\mathscr{B}_{0 *}^{p}\left(U^{n}\right)$. Now the proof of Lemma 2.3 is completed.

Lemma 2.4. Let $\phi=\left(\phi_{1}, \phi_{2}, \ldots, \phi_{n}\right)$ be a holomorphic self-map of $U^{n}$. Then $C_{\phi}: \mathscr{R}_{0 *}^{p}$ $\left(U^{n}\right)\left(\mathscr{B}_{0}^{p}\left(U^{n}\right)\right) \rightarrow \mathscr{B}_{0 *}^{q}\left(U^{n}\right)$ is bounded if and only if $\phi_{l} \in \mathscr{B}_{0 *}^{q}\left(U^{n}\right)$ for every $l=1,2, \ldots, n$ and (2.8) holds.

Proof. If $C_{\phi}: \mathscr{B}_{0 *}^{p}\left(U^{n}\right)\left(\mathscr{B}_{0}^{p}\left(U^{n}\right)\right) \rightarrow \mathscr{B}_{0 *}^{q}\left(U^{n}\right)$ is bounded, it is clear that, for every $l=$ $1,2, \ldots, n, f_{l}(z)=z_{l} \in \mathscr{B}_{0}^{p}\left(U^{n}\right) \subset \mathscr{B}_{0 *}^{q}\left(U^{n}\right)$, so $C_{\phi} f_{l}=\phi_{l} \in \mathscr{B}_{0 *}^{q}\left(U^{n}\right)$. Furthermore, (2.12) holds by Lemma 2.3 .

In order to prove the converse, we first prove that if $\phi_{l} \in \mathscr{B}_{0 *}^{q}\left(U^{n}\right)$, for every $l=$ $1,2, \ldots, n$, then $f \circ \phi \in \mathscr{B}_{0 *}^{q}\left(U^{n}\right)$ for any $f \in \mathscr{B}_{0 *}^{p}\left(U^{n}\right)$.

Without loss of generality, we prove this result when $n=2$.

For any sequence $\left\{z^{j}=\left(z_{1}^{j}, z_{2}^{j}\right)\right\} \subset U^{n}$ with $z^{j} \rightarrow \partial^{*} U^{n}$ as $j \rightarrow \infty$, then

$$
\left|z_{1}^{j}\right| \longrightarrow 1, \quad\left|z_{2}^{j}\right| \longrightarrow 1
$$

Since $\left|\phi_{1}\left(z^{j}\right)\right|<1$ and $\left|\phi_{2}\left(z^{j}\right)\right|<1$, there exists a subsequence $\left\{z^{j_{s}}\right\}$ in $\left\{z^{j}\right\}$ such that

$$
\left|\phi_{1}\left(z^{j_{s}}\right)\right| \longrightarrow \rho_{1}, \quad\left|\phi_{2}\left(z^{j_{s}}\right)\right| \longrightarrow \rho_{2}
$$

as $s \rightarrow \infty$. 
8 Essential norm of composition operators

It is clear that $0 \leq \rho_{1}, \rho_{2} \leq 1$. Then for $k=1,2$, we have

$$
\begin{aligned}
& \left|\frac{\partial(f \circ \phi)}{\partial z_{k}}\left(z^{j_{s}}\right)\right|\left(1-\left|z_{k}^{j_{s}}\right|^{2}\right)^{q} \\
& \leq\left|\frac{\partial f}{\partial w_{1}}\left(\phi\left(z^{j_{s}}\right)\right)\right|\left|\frac{\partial \phi_{1}}{\partial z_{k}}\left(z^{j_{s}}\right)\right|\left(1-\left|z_{k}^{j_{s}}\right|^{2}\right)^{q} \\
& \quad+\left|\frac{\partial f}{\partial w_{2}}\left(\phi\left(z^{j_{s}}\right)\right)\right|\left|\frac{\partial \phi_{2}}{\partial z_{k}}\left(z^{j_{s}}\right)\right|\left(1-\left|z_{k}^{j_{s}}\right|^{2}\right)^{q} \\
& =\left|\frac{\partial f}{\partial w_{1}}\left(\phi\left(z^{j_{s}}\right)\right)\right|\left(1-\left|\phi_{1}\left(z^{j_{s}}\right)\right|^{2}\right)^{p}\left|\frac{\partial \phi_{1}}{\partial z_{k}}\left(z^{j_{s}}\right)\right| \frac{\left(1-\left|z_{k}^{j_{s}}\right|^{2}\right)^{q}}{\left(1-\left|\phi_{1}\left(z^{j_{s}}\right)\right|^{2}\right)^{p}} \\
& \quad+\left|\frac{\partial f}{\partial w_{2}}\left(\phi\left(z^{j_{s}}\right)\right)\right|\left(1-\left|\phi_{2}\left(z^{j_{s}}\right)\right|^{2}\right)^{p}\left|\frac{\partial \phi_{2}}{\partial z_{k}}\left(z^{j_{s}}\right)\right| \frac{\left(1-\left|z_{k}^{j_{s}}\right|^{2}\right)^{q}}{\left(1-\left|\phi_{2}\left(z^{j_{s}}\right)\right|^{2}\right)^{p}} .
\end{aligned}
$$

Now we prove the left-hand side of $(2.16) \rightarrow 0$ as $s \rightarrow \infty$ according to four cases.

Case 1. If $\rho_{1}<1$ and $\rho_{2}<1$, there exist $r_{1}$ and $r_{2}$ such that $\rho_{1}<r_{1}<1$ and $\rho_{2}<r_{2}<1$, so as $j$ is large enough, $\left|\phi_{1}\left(z^{j_{s}}\right)\right| \leq r_{1}$ and $\left|\phi_{2}\left(z^{j_{s}}\right)\right| \leq r_{2}$.

Since $\phi_{1}, \phi_{2} \in \mathscr{B}_{0 *}^{q}\left(U^{n}\right)$, by (2.16), we get

$$
\begin{aligned}
\left|\frac{\partial(f \circ \phi)}{\partial z_{k}}\left(z^{j_{s}}\right)\right|\left(1-\left|z_{k}^{j_{s}}\right|^{2}\right)^{q} \leq & \|f\|_{p} \frac{1}{\left(1-r_{1}^{2}\right)^{p}}\left|\frac{\partial \phi_{1}}{\partial z_{k}}\left(z^{j_{s}}\right)\right|\left(1-\left|z_{k}^{j_{s}}\right|^{2}\right)^{q} \\
& +\|f\|_{p} \frac{1}{\left(1-r_{2}^{2}\right)^{p}}\left|\frac{\partial \phi_{2}}{\partial z_{k}}\left(z^{j_{s}}\right)\right|\left(1-\left|z_{k}^{j_{s}}\right|^{2}\right)^{q} \longrightarrow 0
\end{aligned}
$$

as $s \rightarrow \infty$.

Case 2. If $\rho_{1}=1$ and $\rho_{2}=1$, then $\phi\left(z^{j_{s}}\right) \rightarrow \partial^{*} U^{n}$, by $(2.8)$ and, since $f \in \mathscr{B}_{0 *}^{p}\left(U^{n}\right),(2.16)$ yields that

$$
\begin{aligned}
& \left|\frac{\partial(f \circ \phi)}{\partial z_{k}}\left(z^{j_{s}}\right)\right|\left(1-\left|z_{k}^{j_{s}}\right|^{2}\right)^{q} \\
& \quad \leq C\left|\frac{\partial f}{\partial w_{1}}\left(\phi\left(z^{j_{s}}\right)\right)\right|\left(1-\left|\phi_{1}\left(z^{j_{s}}\right)\right|^{2}\right)^{p}+C\left|\frac{\partial f}{\partial w_{2}}\left(\phi\left(z^{j_{s}}\right)\right)\right|\left(1-\left|\phi_{2}\left(z^{j_{s}}\right)\right|^{2}\right)^{p} \longrightarrow 0
\end{aligned}
$$


Case 3. If $\rho_{1}<1$ and $\rho_{2}=1$, similarly to Case 1 , we can prove that

$$
\begin{aligned}
& \left|\frac{\partial f}{\partial w_{1}}\left(\phi\left(z^{j_{s}}\right)\right)\right|\left(1-\left|\phi_{1}\left(z^{j_{s}}\right)\right|^{2}\right)^{p}\left|\frac{\partial \phi_{1}}{\partial z_{k}}\left(z^{j_{s}}\right)\right| \frac{\left(1-\left|z_{k}^{j_{s}}\right|^{2}\right)^{q}}{\left(1-\left|\phi_{1}\left(z^{j_{s}}\right)\right|^{2}\right)^{p}} \\
& \quad \leq\|f\|_{p} \frac{1}{\left(1-r_{1}^{2}\right)^{p}}\left|\frac{\partial \phi_{1}}{\partial z_{k}}\left(z^{j_{s}}\right)\right| \frac{\left(1-\left|z_{k}^{j_{s}}\right|^{2}\right)^{q}}{\left(1-\left|\phi_{1}\left(z^{j_{s}}\right)\right|^{2}\right)^{p}} \longrightarrow 0
\end{aligned}
$$

as $s \rightarrow \infty$.

On the other hand, for fixed $s$, let $w_{2}^{j_{s}}=\phi_{2}\left(z^{j_{s}}\right)$. Then $\left|w_{2}^{j_{s}}\right|<1$. Denote

$$
F\left(w_{1}\right)=\frac{\partial f}{\partial w_{2}}\left(w_{1}, w_{2}^{j_{s}}\right) .
$$

It is clear that $F\left(w_{1}\right)$ is holomorphic on $\left|w_{1}\right|<1$. Choosing $R_{j_{s}} \rightarrow 1$ with $r_{1} \leq R_{j_{s}}<1$. $\left|\phi_{1}\left(z^{j_{s}}\right)\right| \leq r_{1}$, so

$$
\left|F\left(\phi_{1}\left(z^{j_{s}}\right)\right)\right| \leq \max _{\left|w_{1}\right| \leq r_{1}}\left|F\left(w_{1}\right)\right| \leq \max _{\left|w_{1}\right| \leq R_{j_{s}}}\left|F\left(w_{1}\right)\right|=\max _{\left|w_{1}\right|=R_{j_{s}}}\left|F\left(w_{1}\right)\right|=\left|F\left(w_{1}^{j_{s}}\right)\right|,
$$

where $w_{1}^{j_{s}}$ is a point of modulus $R_{j_{s}}$ where maximum of $F\left(w_{1}\right)$ is attained. This means that $\left|\left(\partial f / \partial w_{2}\right)\left(\phi_{1}\left(z^{j_{s}}\right), \phi_{2}\left(z^{j_{s}}\right)\right)\right| \leq\left|\left(\partial f / \partial w_{2}\right)\left(w_{1}^{j_{s}}, w_{2}^{j_{s}}\right)\right|$. Since $\left|w_{1}^{j_{s}}\right| \rightarrow 1,\left|w_{2}^{j_{s}}\right| \rightarrow \rho_{2}=1$ and $f \in \mathscr{B}_{0 *}^{p}\left(U^{n}\right)$,

$$
\left|\frac{\partial f}{\partial w_{2}}\left(w_{1}^{j_{s}}, w_{2}^{j_{s}}\right)\right|\left(1-\left|w_{2}^{j_{s}}\right|^{2}\right)^{p} \longrightarrow 0
$$

as $s \rightarrow \infty$, so by $(2.8)$,

$$
\begin{aligned}
& \left|\frac{\partial f}{\partial w_{2}}\left(\phi\left(z^{j_{s}}\right)\right)\right|\left(1-\left|\phi_{2}\left(z^{j_{s}}\right)\right|^{2}\right)^{p}\left|\frac{\partial \phi_{2}}{\partial z_{k}}\left(z^{j_{s}}\right)\right| \frac{\left(1-\left|z_{k}^{j_{s}}\right|^{2}\right)^{q}}{\left(1-\left|\phi_{2}\left(z^{j_{s}}\right)\right|^{2}\right)^{p}} \\
& \quad \leq C\left|\frac{\partial f}{\partial w_{2}}\left(w_{1}^{j_{s}}, w_{2}^{j_{s}}\right)\right|\left(1-\left|w_{2}^{j_{s}}\right|^{2}\right)^{p} \longrightarrow 0
\end{aligned}
$$

as $s \rightarrow \infty$.

By (2.19) and (2.23), (2.16) yields

$$
\left|\frac{\partial(f \circ \phi)}{\partial z_{k}}\left(z^{j_{s}}\right)\right|\left(1-\left|z_{k}^{j_{s}}\right|^{2}\right)^{q} \longrightarrow 0,
$$

as $s \rightarrow \infty$.

Case 4. If $\rho_{1}=1$ and $\rho_{2}<1$, similarly to Case 3 , we can prove

$$
\left|\frac{\partial(f \circ \phi)}{\partial z_{k}}\left(z^{j_{s}}\right)\right|\left(1-\left|z_{k}^{j_{s}}\right|^{2}\right)^{q} \longrightarrow 0,
$$

as $s \rightarrow \infty$. 
Combining Cases 1, 2, 3, and 4, we know there exists a subsequence $\left\{z^{j_{s}}\right\}$ in $\left\{z^{j}\right\}$ such that

$$
\left|\frac{\partial(f \circ \phi)}{\partial z_{k}}\left(z^{j_{s}}\right)\right|\left(1-\left|z_{k}^{j_{s}}\right|^{2}\right)^{q} \longrightarrow 0,
$$

as $s \rightarrow \infty$ for $k=1,2$. We claim that

$$
\left|\frac{\partial(f \circ \phi)}{\partial z_{k}}\left(z^{j}\right)\right|\left(1-\left|z_{k}^{j}\right|^{2}\right)^{q} \longrightarrow 0,
$$

as $j \rightarrow \infty$. In fact, if it fails, then there exists a subsequence $\left\{z^{j_{s}}\right\}$ such that

$$
\left|\frac{\partial(f \circ \phi)}{\partial z_{k}}\left(z^{j_{s}}\right)\right|\left(1-\left|z_{k}^{j_{s}}\right|^{2}\right)^{q} \longrightarrow \varepsilon>0
$$

for $k=1$ or 2 . But from the above discussion, we can find a subsequence in $\left\{z^{j_{s}}\right\}$; we still write $\left\{z^{j_{s}}\right\}$ with

$$
\left|\frac{\partial(f \circ \phi)}{\partial z_{k}}\left(z^{j_{s}}\right)\right|\left(1-\left|z_{k}^{j_{s}}\right|^{2}\right)^{q} \longrightarrow 0,
$$

it contradicts with (2.28).

So for any sequence $\left\{z^{j}\right\} \subset U^{n}$ with $z^{j} \rightarrow \partial^{*} U^{n}$ as $j \rightarrow \infty$, we have

$$
\left|\frac{\partial(f \circ \phi)}{\partial z_{k}}\left(z^{j}\right)\right|\left(1-\left|z_{k}^{j}\right|^{2}\right)^{q} \longrightarrow 0
$$

for $k=1$, 2. By (2.8) and Lemma 2.3, it is clear that $f \circ \phi \in \mathscr{B}^{q}\left(U^{n}\right)$, so $f \circ \phi \in \mathscr{B}_{0 *}^{q}\left(U^{n}\right)$.

For any $f \in \mathscr{B}_{0}^{p}\left(U^{n}\right)$. Since $\mathscr{B}_{0}^{p}\left(U^{n}\right) \subset \mathscr{B}_{0 *}^{p}\left(U^{n}\right)$, then $f \circ \phi \in \mathscr{B}_{0 *}^{q}\left(U^{n}\right)$.

By closed graph theorem, we know that

$$
C_{\phi}: \mathscr{B}_{0 *}^{p}\left(U^{n}\right)\left(\mathscr{B}_{0}^{p}\left(U^{n}\right)\right) \longrightarrow \mathscr{B}_{0 *}^{q}\left(U^{n}\right)
$$

is bounded. This ends the proof of Lemma 2.4.

Remark 2.5. For the case $C_{\phi}: \mathscr{B}_{P} P\left(U^{n}\right) \rightarrow \mathscr{B}_{0 *}^{q}\left(U^{n}\right)$, the necessity also holds, but we cannot guarantee that the sufficiency holds because we cannot be sure that $C_{\phi} f \in \mathscr{B}_{0 *}^{q}\left(U^{n}\right)$ for all $f \in \mathscr{M}^{p}\left(U^{n}\right)$.

Lemma 2.6. Let $\phi=\left(\phi_{1}, \phi_{2}, \ldots, \phi_{n}\right)$ be a holomorphic self-map of $U^{n}$. Then

$$
C_{\phi}: \mathscr{B}_{0}^{p}\left(U^{n}\right) \longrightarrow \mathscr{B}_{0}^{q}\left(U^{n}\right)
$$

is bounded if and only if $\phi^{\gamma} \in \mathscr{B}_{0}^{q}\left(U^{n}\right)$ for every multiindex $\gamma$, and (2.8) holds.

Proof (sufficiency). From (2.8) and by Lemma 2.3 we know that $C_{\phi}: \mathscr{B}^{P}\left(U^{n}\right) \rightarrow \mathscr{R}^{q}\left(U^{n}\right)$ is bounded, in particular

$$
\left\|C_{\phi} f\right\|_{q} \leq\left\|C_{\phi}\right\|_{\mathscr{B}^{p}\left(U^{n}\right) \rightarrow \Re^{q}\left(U^{n}\right)}\|f\|_{p}, \quad \forall f \in \mathscr{B}_{0}^{p}\left(U^{n}\right) .
$$


The boundedness of $C_{\phi}: \mathscr{B}_{0}^{p}\left(U^{n}\right) \rightarrow \mathscr{B}_{0}^{q}\left(U^{n}\right)$ directly follows, if we prove $C_{\phi} f \in \mathscr{B}_{0}^{q}\left(U^{n}\right)$ whenever $f \in \mathscr{B}_{0}^{p}\left(U^{n}\right)$. So, let $f \in \mathscr{B}_{0}^{p}\left(U^{n}\right)$. By the definition of $\mathscr{B}_{0}^{p}\left(U^{n}\right)$ it follows that for every $\varepsilon>0$ there is a polynomial $p_{\varepsilon}$ such that $\left\|f-p_{\varepsilon}\right\|_{p}<\varepsilon$. Hence

$$
\left\|C_{\phi} f-C_{\phi} p_{\varepsilon}\right\|_{q} \leq\left\|C_{\phi}\right\|_{\mathscr{B} p\left(U^{n}\right) \rightarrow \mathscr{B} q\left(U^{n}\right)}\left\|f-p_{\varepsilon}\right\|_{p}<\varepsilon\left\|C_{\phi}\right\|_{\mathscr{B} p\left(U^{n}\right) \rightarrow \Re^{q}\left(U^{n}\right)} .
$$

Since $\phi^{\gamma} \in \mathscr{B}_{0}^{q}\left(U^{n}\right)$ for every multiindex $\gamma$, we obtain $C_{\phi} p_{\varepsilon} \in \mathscr{B}_{0}^{q}\left(U^{n}\right)$. From this and (2.34) the result follows.

If $C_{\phi}: \mathscr{B}_{0}^{p}\left(U^{n}\right) \rightarrow \mathscr{P}_{0}^{q}\left(U^{n}\right)$ is bounded, then (2.8) can be proved as in Lemma 2.3 , since the test functions appearing there belong to $\mathscr{B}_{0}^{p}\left(U^{n}\right)$. Since the polynomials $z^{\gamma} \in \mathscr{B}_{0}^{p}\left(U^{n}\right)$ for every multiindex $\gamma$, we get $C_{\phi} z^{\gamma} \in \mathscr{B}_{0}^{q}\left(U^{n}\right)$, as desired.

Remark 2.7. For the case $C_{\phi}: \mathscr{B}^{p}\left(U^{n}\right)\left(\mathscr{B}_{0 *}^{p}\left(U^{n}\right)\right) \rightarrow \mathscr{B}_{0}^{q}\left(U^{n}\right)$, in analogy to Remark 2.5, the necessity also holds, but we cannot guarantee that the sufficiency holds.

LEMMA 2.8. If $\left\{f_{k}\right\}$ is a bounded sequence in $\mathscr{B}^{P}\left(U^{n}\right)$, then there exists a subsequence $\left\{f_{k_{l}}\right\}$ of $\left\{f_{k}\right\}$ which converges uniformly on compact subsets of $U^{n}$ to a holomorphic function $f \in$ $\mathscr{H}^{P}\left(U^{n}\right)$.

Proof. Let $\left\{f_{k}\right\}$ be a bounded sequence in $\mathscr{B}^{P}\left(U^{n}\right)$ with $\left\|f_{k}\right\|_{p} \leq C$. By Lemma 2.1, $\left\{f_{j}\right\}$ is uniformly bounded on compact subsets of $U^{n}$ and hence normal by Montel's theorem. So we may extract a subsequence $\left\{f_{j_{k}}\right\}$ which converges uniformly on compact subsets of $U^{n}$ to a holomorphic function $f$. It follows that $\partial f_{j_{k}} / \partial z_{l} \rightarrow \partial f / \partial z_{l}$ for each $l \in\{1,2, \ldots, n\}$, so

$$
\sum_{l=1}^{n}\left|\frac{\partial f}{\partial z_{l}}\right|\left(1-\left|z_{l}\right|^{2}\right)^{p}=\lim _{k \rightarrow \infty} \sum_{l=1}^{n}\left|\frac{\partial f_{j_{k}}}{\partial z_{l}}\right|\left(1-\left|z_{l}\right|^{2}\right)^{p} \leq \sup _{k}\left\|f_{j_{k}}\right\|_{p} \leq C
$$

which implies $f \in \mathscr{M}^{P}\left(U^{n}\right)$. The Lemma is proved.

Lemma 2.9. Let $\Omega$ be a domain in $\mathbb{C}^{n}, f \in H(\Omega)$. If a compact set $K$ and its neighborhood $G$ satisfy $K \subset G \subset \bar{G} \subset \Omega$ and $\rho=\operatorname{dist}(K, \partial G)>0$, then

$$
\sup _{z \in K}\left|\frac{\partial f}{\partial z_{j}}(z)\right| \leq \frac{\sqrt{n}}{\rho} \sup _{z \in G}|f(z)| \text {. }
$$

Proof. For any $a \in K$, the polydisc

$$
P_{a}=\left\{\left(z_{1}, \ldots, z_{n}\right) \in \mathbb{C}^{n}:\left|z_{j}-a_{j}\right|<\frac{\rho}{\sqrt{n}}, j=1, \ldots, n\right\}
$$

is contained in G. By Cauchy's inequality,

$$
\left|\frac{\partial f}{\partial z_{j}}(a)\right| \leq \frac{\sqrt{n}}{\rho} \sup _{z \in \partial^{*} P_{a}}|f(z)| \leq \frac{\sqrt{n}}{\rho} \sup _{z \in G}|f(z)| .
$$

Taking the supremum for $a$ over $K$ gives the desired inequality. 


\section{The proof of Theorem 1.1}

Now we turn to the proof of Theorem 1.1. In the following, we are dealing with the case for $C_{\phi}: \mathscr{B}^{P}\left(U^{n}\right) \rightarrow \mathscr{B}^{q}\left(U^{n}\right)$, but if we note that the test functions $f_{m}$ introduced below belong to $\mathscr{B}_{0}^{p}\left(U^{n}\right) \subset \mathscr{B}_{0 *}^{p}\left(U^{n}\right) \subset \mathscr{B}^{p}\left(U^{n}\right)$, the results in Theorem 1.1 also hold with minor modifications for the other cases.

We begin by proving the lower estimate. It is clear that $\left\{m^{p-1} z_{1}^{m}\right\} \subset \mathscr{B}_{0}^{p}\left(U^{n}\right) \subset$ $\mathscr{B}_{0 *}\left(U^{n}\right) \subset \mathscr{B}_{(}\left(U^{n}\right)$ for $m=1,2, \ldots$, and this sequence converges to zero uniformly on compact subsets of the unit polydisc $U^{n}$. Furthermore

$$
\left\|m^{p-1} z_{1}^{m}\right\|_{p}=\sup _{z \in U^{n}}\left(1-\left|z_{1}\right|^{2}\right)^{p} m^{p}\left|z_{1}\right|^{m-1}
$$

Let $p(x)=m^{p}\left(1-x^{2}\right)^{p} x^{m-1}$, then

$$
p^{\prime}(x)=-m^{p} x^{m-2}\left(1-x^{2}\right)^{p-1}\left[(2 p+m-1) x^{2}-(m-1)\right]
$$

so

$$
\begin{array}{ll}
p^{\prime}(x) \leq 0 & \text { for } x \in[\sqrt{(m-1) /(2 p+m-1)}, 1] \\
p^{\prime}(x) \geq 0 & \text { for } x \in[0, \sqrt{(m-1) /(2 p+m-1)}] .
\end{array}
$$

That is, $p(x)$ is a decreasing function for $x \in[\sqrt{(m-1) /(2 p+m-1)}, 1]$ and $p(x)$ is an increasing function for $x \in[0, \sqrt{(m-1) /(2 p+m-1)}]$. Hence

$$
\max _{x \in[0,1]} p(x)=p\left(\sqrt{\frac{m-1}{2 p+m-1}}\right) .
$$

It follows from (3.1) that

$$
\left\|m^{p-1} z_{1}^{m}\right\|_{p}=p\left(\sqrt{\frac{m-1}{2 p+m-1}}\right)=\left(\frac{2 p}{2 p+m-1}\right)^{p} m^{p}\left(\frac{m-1}{2 p+m-1}\right)^{(m-1) / 2} \longrightarrow\left(\frac{2 p}{e}\right)^{p},
$$

as $m \rightarrow \infty$.

Therefore, the sequence $\left\{m^{p-1} z_{1}^{m}\right\}_{m \geq 2}$ is bounded away from zero. Now we consider the normalized sequence $\left\{f_{m}=m^{p-1} z_{1}^{m} /\left\|m^{p-1} z_{1}^{m}\right\|_{p}\right\}$ which also tends to zero uniformly on compact subsets of $U^{n}$. For each $m \geq 2$, we define

$$
A_{m}=\left\{z=\left(z_{1}, \ldots, z_{n}\right) \in U^{n}: r_{m} \leq\left|z_{1}\right| \leq r_{m+1}\right\},
$$


where $r_{m}=\sqrt{(m-1) /(2 p+m-1)}$. So

$$
\begin{aligned}
\min _{A_{m}} & \sum_{l=1}^{n}\left\{\left|\frac{\partial f_{m}}{\partial z_{l}}(z)\right|\left(1-\left|z_{l}\right|^{2}\right)^{p}\right\} \\
& =\min _{A_{m}}\left|\frac{\partial f_{m}}{\partial z_{1}}\right|\left(1-\left|z_{1}\right|^{2}\right)^{p}=\frac{\left(1-r_{m+1}^{2}\right)^{p} m^{p} r_{m+1}^{m-1}}{\left\|m^{p-1} z_{1}^{m}\right\|_{p}} \\
& =\left(\frac{2 p+m-1}{2 p+m}\right)\left(\frac{m(2 p+m-1)}{(m-1)(2 p+m)}\right)^{((m-1) / 2)}=c_{m} .
\end{aligned}
$$

It is easy to show that $c_{m}$ tends to 1 as $m \rightarrow \infty$. For the moment fix any compact operator $K: \mathscr{B}^{p}\left(U^{n}\right) \rightarrow \mathscr{B}^{q}\left(U^{n}\right)$. The uniform convergence on compact subsets of the sequence $\left\{f_{m}\right\}$ to zero and the compactness of $K$ imply that $\left\|K f_{m}\right\|_{q} \rightarrow 0$. It is easy to show that if a bounded sequence that is contained in $\mathscr{B}_{0 *}^{p}\left(U^{n}\right)$ converges uniformly on compact subsets of $U^{n}$, then it also converges weakly to zero in $\mathscr{B}_{0 *}^{p}\left(U^{n}\right)$ as well as in $\mathscr{B} P\left(U^{n}\right)$. Since $\left\|f_{m}\right\|_{p}=1$, we have

$$
\begin{aligned}
\left\|C_{\phi}-K\right\| & \geq \limsup _{m}\left\|\left(C_{\phi}-K\right) f_{m}\right\|_{q} \\
& \geq \limsup _{m}\left(\left\|C_{\phi} f_{m}\right\|_{q}-\left\|K f_{m}\right\|_{q}\right)=\limsup _{m}\left\|C_{\phi} f_{m}\right\|_{q} \\
& \geq \limsup _{m} \sup _{z \in U^{n}} \sum_{k=1}^{n}\left\{\left|\frac{\partial\left(f_{m} \circ \phi\right)}{\partial z_{k}}(z)\right|\left(1-\left|z_{k}\right|^{2}\right)^{q}\right\} \\
& =\limsup _{m} \sup _{z \in U^{n}} \sum_{k=1}^{n}\left|\frac{\partial f_{m}}{\partial w_{1}}(\phi(z))\right|\left|\frac{\partial \phi_{1}}{\partial z_{k}}(z)\right|\left(1-\left|z_{k}\right|^{2}\right)^{q} \\
& =\limsup _{m} \sup _{z \in U^{n}} \sum_{k=1}^{n}\left|\frac{\partial \phi_{1}}{\partial z_{k}}(z)\right| \frac{\left(1-\left|z_{k}\right|^{2}\right)^{q}}{\left(1-\left|\phi_{1}(z)\right|^{2}\right)^{p}}\left|\frac{\partial f_{m}}{\partial w_{1}}(\phi(z))\right|\left(1-\left|\phi_{1}(z)\right|^{2}\right)^{p} \\
\geq & \limsup _{m} \sup _{\phi(z) \in A_{m}} \sum_{k=1}^{n}\left|\frac{\partial \phi_{1}}{\partial z_{k}}(z)\right| \frac{\left(1-\left|z_{k}\right|^{2}\right)^{q}}{\left(1-\left|\phi_{1}(z)\right|^{2}\right)^{p}}\left|\frac{\partial f_{m}}{\partial w_{1}}(\phi(z))\right|\left(1-\left|\phi_{1}(z)\right|^{2}\right)^{p} \\
& \geq \limsup _{m} \sup _{\phi(z) \in A_{m}} \sum_{k=1}^{n}\left|\frac{\partial \phi_{1}}{\partial z_{k}}(z)\right| \frac{\left(1-\left|z_{k}\right|^{2}\right)^{q}}{\left(1-\left|\phi_{1}(z)\right|^{2}\right)^{p}} \\
& \times \liminf _{\phi(z) \in A_{m}} \min _{\frac{\partial w_{m}}{\partial w_{1}}(\phi(z)) \mid\left(1-\left|\phi_{1}(z)\right|^{2}\right)^{p}}
\end{aligned}
$$




$$
\begin{aligned}
& \geq \limsup \sup _{\phi(z) \in A_{m}} \sum_{k=1}^{n}\left|\frac{\partial \phi_{1}}{\partial z_{k}}(z)\right| \frac{\left(1-\left|z_{k}\right|^{2}\right)^{q}}{\left(1-\left|\phi_{1}(z)\right|^{2}\right)^{p}} \liminf _{m} c_{m} \\
& \geq \limsup \sup _{\phi} \sum_{\phi(z) \in A_{m}}^{n}\left|\frac{\partial \phi_{1}}{\partial z_{k}}(z)\right| \frac{\left(1-\left|z_{k}\right|^{2}\right)^{q}}{\left(1-\left|\phi_{1}(z)\right|^{2}\right)^{p}} .
\end{aligned}
$$

So

$$
\begin{aligned}
\left\|C_{\phi}\right\|_{e} & =\inf \left\{\left\|C_{\phi}-K\right\|: K \text { is compact }\right\} \\
& \geq \lim \sup _{m} \sup _{\phi(z) \in A_{m}} \sum_{k=1}^{n}\left|\frac{\partial \phi_{1}}{\partial z_{k}}(z)\right| \frac{\left(1-\left|z_{k}\right|^{2}\right)^{q}}{\left(1-\left|\phi_{1}(z)\right|^{2}\right)^{p}} .
\end{aligned}
$$

For each $l=1,2, \ldots, n$, define

$$
a_{l}=\lim _{\delta \rightarrow 0} \sup _{\operatorname{dist}\left(\phi(z), \partial U^{n}\right)<\delta} \sum_{k=1}^{n}\left|\frac{\partial \phi_{l}}{\partial z_{k}}(z)\right| \frac{\left(1-\left|z_{k}\right|^{2}\right)^{q}}{\left(1-\left|\phi_{l}(z)\right|^{2}\right)^{p}} .
$$

For any $\varepsilon>0,(3.10)$ shows that there exists a $\delta_{0}$ with $0<\delta_{0}<1$, such that

$$
\sum_{k=1}^{n}\left|\frac{\partial \phi_{l}}{\partial z_{k}}(z)\right| \frac{\left(1-\left|z_{k}\right|^{2}\right)^{q}}{\left(1-\left|\phi_{l}(z)\right|^{2}\right)^{p}}>a_{l}-\varepsilon
$$

whenever $\operatorname{dist}\left(\phi(z), \partial U^{n}\right)<\delta_{0}$ and $l=1,2, \ldots, n$.

Since $r_{m} \rightarrow 1$ as $m \rightarrow \infty$, we may choose $m$ large enough so that $r_{m}>1-\delta_{0}$. If $\phi(z) \in$ $A_{m}, r_{m} \leq\left|\phi_{1}(z)\right| \leq r_{m+1}$, so $1-r_{m+1}<1-\left|\phi_{1}(z)\right|<1-r_{m}<\delta_{0}$; hence $\operatorname{dist}\left(\phi_{1}(z), \partial U\right)<$ $\delta_{0}$. There exists $w_{1}$ with $\left|w_{1}\right|=1$ such that $\operatorname{dist}\left(\phi_{1}(z), w_{1}\right)=\operatorname{dist}\left(\phi_{1}(z), \partial U\right)<\delta_{0}$.

Let $w=\left(w_{1}, \phi_{2}(z), \ldots, \phi_{n}(z)\right) \in \partial U^{n}$. Then

$$
\operatorname{dist}\left(\phi(z), \partial U^{n}\right) \leq \operatorname{dist}(\phi(z), w)=\operatorname{dist}\left(\phi_{1}(z), w_{1}\right)<\delta_{0} .
$$

By (3.11), (3.9) implies that

$$
\left\|C_{\phi}\right\|_{e} \geq a_{1}-\varepsilon
$$

Similarly, if we choose $g_{m}(z)=m^{p-1} z_{l}^{m} /\left\|m^{p-1} z_{l}^{m}\right\|$, we have

$$
\left\|C_{\phi}\right\|_{e} \geq a_{l}-\varepsilon
$$


for every $l=2 \ldots, n$. So

$$
\begin{aligned}
\left\|C_{\phi}\right\|_{e} & \geq \frac{1}{n} \sum_{l=1}^{n}\left(a_{l}-\varepsilon\right) \\
& =\frac{1}{n} \sum_{l=1}^{n}\left(\lim _{\delta \rightarrow 0} \sup _{\operatorname{dist}\left(\phi(z), \partial U^{n}\right)<\delta} \sum_{k=1}^{n}\left|\frac{\partial \phi_{l}}{\partial z_{k}}(z)\right| \frac{\left(1-\left|z_{k}\right|^{2}\right)^{q}}{\left(1-\left|\phi_{l}(z)\right|^{2}\right)^{p}}-\varepsilon\right) \\
& \geq \frac{1}{n} \lim _{\delta \rightarrow 0} \sup _{\operatorname{dist}\left(\phi(z), \partial U^{n}\right)<\delta} \sum_{k, l=1}^{n}\left|\frac{\partial \phi_{l}}{\partial z_{k}}(z)\right| \frac{\left(1-\left|z_{k}\right|^{2}\right)^{q}}{\left(1-\left|\phi_{l}(z)\right|^{2}\right)^{p}}-\varepsilon .
\end{aligned}
$$

Let $\varepsilon \rightarrow 0$, the low estimate follows.

To obtain the upper estimate we first prove the following proposition.

Proposition 3.1. Let $\phi=\left(\phi_{1}, \ldots, \phi_{n}\right)$ be a holomorphic self-map of $U^{n}$. Then for $m \geq 2$, the operator $K_{m}$ on $H\left(U^{n}\right)$ defined by $K_{m} f(z)=f(((m-1) / m) z)$ has the following properties. For each $f \in H\left(U^{n}\right)$,

(i) $K_{m} f \in \mathscr{B}_{0}^{p}\left(U^{n}\right) \subset \mathscr{B}_{0 *}^{p}\left(U^{n}\right) \subset \mathscr{B}^{p}\left(U^{n}\right)$;

(ii) if $C_{\phi}: \mathscr{B} P\left(U^{n}\right) \rightarrow \mathscr{B}^{q}\left(U^{n}\right)$ is bounded, then $C_{\phi} K_{m} f \in \mathscr{B}^{q}\left(U^{n}\right)$;

(iii) for fixed $m$, the operator $K_{m}$ is compact on $\mathscr{B}^{P}\left(U^{n}\right)$;

(iv) if $C_{\phi}: \mathscr{B} P\left(U^{n}\right) \rightarrow \mathscr{B}^{q}\left(U^{n}\right)$ is bounded, then $C_{\phi} K_{m} f \in \mathscr{B}^{q}\left(U^{n}\right)$ is compact;

(v) $\left\|I-K_{m}\right\| \leq 2$;

(vi) $\left(I-K_{m}\right) f$ converges to zero uniformly on compacta in $U^{n}$.

Proof. (i) Let $f \in H\left(U^{n}\right), r_{m}=(m-1) / m$, and $f_{m}(z)=K_{m} f(z)=f\left(r_{m} z\right)$. First note that

$$
\begin{aligned}
\left\|f_{m}\right\|_{p} & =|f(0)|+\sup _{z \in U^{n}} \sum_{k=1}^{n} r_{m}\left|\frac{\partial f}{\partial z_{k}}\left(r_{m} z\right)\right|\left(1-\left|z_{k}\right|^{2}\right)^{p} \\
& \leq|f(0)|+\sup _{z \in U^{n}} \sum_{k=1}^{n}\left|\frac{\partial f}{\partial z_{k}}\left(r_{m} z\right)\right|\left(1-\left|r_{m} z_{k}\right|^{2}\right)^{p} \leq\|f\|_{p} .
\end{aligned}
$$

On the other hand, $f_{m} \in H\left(\left(1 / r_{m}\right) U^{n}\right)$, and observe that $\left(2 /\left(1+r_{m}\right)\right) \overline{U^{n}} \subset\left(1 / r_{m}\right) U^{n}$ which implies that for fixed $m$, corresponding to each $j=1,2, \ldots$, there is a polynomial $P_{m}^{(j)}$ such that

$$
\sup _{z \in\left(2 /\left(1+r_{m}\right)\right) \overline{U^{n}}}\left|f_{m}(z)-P_{m}^{(j)}(z)\right|<\left(1-r_{m}\right)^{2} \frac{1}{j} .
$$

Let $K=\overline{U^{n}}, G=\left(2 /\left(1+r_{m}\right)\right) U^{n}, \Omega=\left(1 / r_{m}\right) U^{n}$, then $K \subset G \subset \bar{G} \subset \Omega$ and $\rho=$ $\operatorname{dist}(K, \partial G)=\left(1-r_{m}\right) /\left(1+r_{m}\right)>0$, so for all $w \in U^{n}, k \in\{1, \ldots, n\}$, it follows from 
Lemma 2.9 that

$$
\begin{aligned}
\left|\frac{\partial\left(f_{m}-P_{m}^{(j)}\right)}{\partial w_{k}}(w)\right| & \leq \sup _{w \in K}\left|\frac{\partial\left(f_{m}-P_{m}^{(j)}\right)}{\partial w_{k}}(w)\right| \\
& \leq \frac{\sqrt{n}\left(1+r_{m}\right)}{1-r_{m}} \sup _{w \in G}\left|f_{m}(w)-P_{m}^{(j)}(w)\right| \\
& \leq \frac{\sqrt{n}\left(1+r_{m}\right)}{1-r_{m}}\left(1-r_{m}^{2}\right) \frac{1}{j} \leq 4 \sqrt{n} \frac{1}{j} .
\end{aligned}
$$

Therefore

$$
\sum_{k=1}^{n}\left|\frac{\partial\left(f_{m}-P_{m}^{(j)}\right)}{\partial w_{k}}(w)\right|\left(1-\left|w_{k}\right|^{2}\right)^{p} \leq 4 n \sqrt{n} \frac{1}{j} \longrightarrow 0
$$

as $j \rightarrow \infty$, that is,

$$
\left\|f_{m}-P_{m}^{(j)}\right\|_{\mathscr{B}^{p}}=\left|f_{m}(0)-P_{m}^{(j)}(0)\right|+\sup _{w \in U^{n}} \sum_{k=1}^{n}\left|\frac{\partial\left(f_{m}-P_{m}^{(j)}\right)}{\partial w_{k}}(w)\right|\left(1-\left|w_{k}\right|^{p}\right)^{p} \longrightarrow 0 .
$$

$P_{m}^{(j)}(w) \in \mathscr{B}_{0}^{p}\left(U^{n}\right)$ implies that $f_{m} \in \mathscr{B}_{0}^{p}\left(U^{n}\right)$.

(ii) follows immediately from (i).

(iii) For any sequence $\left\{f_{j}\right\} \subset \mathscr{B}^{p}\left(U^{n}\right)$ with $\left\|f_{j}\right\|_{p} \leq M$, by (i), $\left\{K_{m} f_{j}\right\} \in \mathscr{B}_{0}^{p}\left(U^{n}\right)$. By Lemma 2.8, there is a subsequence $\left\{f_{j_{s}}\right\}$ of $\left\{f_{j}\right\}$ which converges uniformly on compact subsets of $U^{n}$ to a holomorphic function $f \in \mathscr{B}^{p}\left(U^{n}\right)$ and $\|f\|_{p} \leq M$. The sequence $\left\{\partial f_{j_{s}} / \partial z_{i}\right\}, i=1,2, \ldots, n$, also converges uniformly on compact subsets of $U^{n}$ to the holomorphic function $\partial f / \partial z_{i}$. So as $s$ is large enough, for any $w \in E=\{((m-1) / m) z: z \in$ $\left.\overline{U^{n}}\right\} \subset U^{n}$,

$$
\left|\frac{\partial\left(f_{j_{s}}-f\right)}{\partial w_{l}}(w)\right|<\varepsilon
$$

for every $l=1,2, \ldots, n$. So

$$
\begin{aligned}
\left\|K_{m} f_{j_{s}}-K_{m} f\right\|_{p}= & \left\|f_{j_{s}}\left(\frac{m-1}{m} z\right)-f\left(\frac{m-1}{m} z\right)\right\|_{p} \\
= & \sup _{z \in U^{n}} \sum_{k=1}^{n}\left\{\left|\frac{\partial\left[\left(f_{j_{s}}-f\right)(((m-1) / m) z)\right]}{\partial z_{k}}\right|\left(1-\left|z_{k}\right|^{2}\right)^{p}\right\} \\
& +\left|f_{j_{s}}(0)-f(0)\right|
\end{aligned}
$$




$$
\begin{aligned}
& \leq \sup _{z \in U^{n}} \sum_{k=1}^{n} \sum_{l=1}^{n}\left|\frac{\partial\left(f_{j_{s}}-f\right)}{\partial w_{l}}\left(\frac{m-1}{m} z\right)\right| \frac{m-1}{m}+\left|f_{j_{s}}(0)-f(0)\right| \\
& \leq n \sup _{w \in E} \frac{m-1}{m} \sum_{l=1}^{n}\left|\frac{\partial\left(f_{j_{s}}-f\right)}{\partial w_{l}}(w)\right|+\left|f_{j_{s}}(0)-f(0)\right| \longrightarrow 0,
\end{aligned}
$$

as $s \rightarrow \infty$. This shows that $\left\{K_{m} f_{j_{s}}\right\}$ converges to $g=K_{m} f \in \mathscr{B}_{0}^{p}\left(U^{n}\right) \subset \mathscr{B}_{0 *}^{p}\left(U^{n}\right) \subset \mathscr{B}^{p}\left(U^{n}\right)$. So $K_{m}$ is compact on $\mathscr{B}^{p}\left(U^{n}\right)$.

(iv) follows immediately from (i) and (iii).

(v) follows from the fact that for any $f \in \mathscr{B}^{P}\left(U^{n}\right),\left(I-K_{m}\right) f(0)=0$, so

$$
\begin{aligned}
\left\|\left(I-K_{m}\right) f\right\|_{p}= & \sup _{z \in U^{n}} \sum_{k=1}^{n}\left|\frac{\partial\left(I-K_{m}\right) f}{\partial z_{k}}(z)\right|\left(1-\left|z_{k}\right|^{2}\right)^{p} \\
= & \sup _{z \in U^{n}} \sum_{k=1}^{n}\left|\frac{\partial f}{\partial z_{k}}(z)-\left(1-\frac{1}{m}\right) \frac{\partial f}{\partial z_{k}}\left(\left(1-\frac{1}{m}\right) z\right)\right|\left(1-\left|z_{k}\right|^{2}\right)^{p} \\
\leq & \sup _{z \in U^{n}} \sum_{k=1}^{n}\left|\frac{\partial f}{\partial z_{k}}(z)\right|\left(1-\left|z_{k}\right|^{2}\right)^{p} \\
& +\left(1-\frac{1}{m}\right) \sup _{z \in U^{n}} \sum_{k=1}^{n}\left|\frac{\partial f}{\partial z_{k}}\left(\left(1-\frac{1}{m}\right) z\right)\right|\left(1-\left|\left(1-\frac{1}{m}\right) z_{k}\right|^{2}\right)^{p} \\
\leq & \|f\|_{p}+\|f\|_{p}=2\|f\|_{p},
\end{aligned}
$$

so $\left\|I-K_{m}\right\| \leq 2$.

(vi) For any compact subset $E \subset U^{n}$, there exists $r, 0<r<1$ such that $E \subset r U^{n} \subset$ $r \overline{U^{n}} \subset U^{n}$. For all $z \in E$,

$$
\begin{aligned}
\left|\left(I-K_{m}\right) f(z)\right| & =\left|f(z)-f_{m}(z)\right|=\left|f(z)-f\left(r_{m} z\right)\right| \\
& \leq \sum_{k=1}^{n} \int_{r_{m}}^{1}\left|\frac{\partial f}{\partial w_{k}}(t z)\right| d t .
\end{aligned}
$$

For $t \in\left[r_{m}, 1\right]$ and $z \in E$, we have $\left|t z_{k}\right|=t\left|z_{k}\right| \leq\left|z_{k}\right|<r, t z \in r U^{n}$, so there exists $M>0$ such that $\left|\left(\partial f / \partial w_{k}\right)(t z)\right| \leq M$ for all $t \in\left[r_{m}, 1\right]$ and $z \in E$. Thus

$$
\left|\left(I-K_{m}\right) f(z)\right| \leq n M\left(1-r_{m}\right) \longrightarrow 0
$$

as $m \rightarrow \infty$, proving the results in Theorem 1.1. 
Let us now return to the proof of the upper estimate. For convenience, we remove the subscript $p$ from $\|f\|_{p}$,

$$
\begin{aligned}
\left\|C_{\phi}\right\|_{e} \leq & \left\|C_{\phi}-C_{\phi} K_{m}\right\|=\left\|C_{\phi}\left(I-K_{m}\right)\right\|=\sup _{\|f\|=1}\left\|C_{\phi}\left(I-K_{m}\right) f\right\|_{q} \\
= & \sup _{\|f\|=1}\left(\sup _{z \in U^{n}} \sum_{k=1}^{n}\left\{\left|\frac{\partial\left(I-K_{m}\right)(f \circ \phi)}{\partial z_{k}}\right|\left(1-\left|z_{k}\right|^{2}\right)^{q}\right\}+\left|\left(I-K_{m}\right) f(\phi(0))\right|\right) \\
\leq & \sup _{\|f\|=1} \sup _{z \in U^{n}} \sum_{k=1}^{n} \sum_{l=1}^{n}\left|\frac{\partial\left(I-K_{m}\right) f}{\partial w_{l}}(\phi(z))\right|\left|\frac{\partial \phi_{l}}{\partial z_{k}}(z)\right|\left(1-\left|z_{k}\right|^{2}\right)^{q} \\
& +\sup _{\|f\|=1}\left|f(\phi(0))-f\left(\frac{m-1}{m} \phi(0)\right)\right| \\
\leq & \sup _{\|f\|=1} \sup _{z \in U^{n}} \sum_{k, l=1}^{n}\left|\frac{\partial \phi_{l}}{\partial z_{k}}(z)\right| \frac{\left(1-\left|z_{k}\right|^{2}\right)^{q}}{\left(1-\left|\phi_{l}(z)\right|^{2}\right)^{p}}\left|\frac{\partial\left(I-K_{m}\right) f}{\partial w_{l}}(\phi(z))\right|\left(1-\left|\phi_{l}(z)\right|^{2}\right)^{p} \\
& +\sup _{\|f\|=1}\left|f(\phi(0))-f\left(\frac{m-1}{m} \phi(0)\right)\right| \cdot
\end{aligned}
$$

Fix $\delta>0$, let $G_{1}=\left\{z \in U^{n}: \operatorname{dist}\left(\phi(z), \partial U^{n}\right)<\delta\right\}, G_{2}=\left\{z \in U^{n}: \operatorname{dist}\left(\phi(z), \partial U^{n}\right) \geq \delta\right\}$, $G=\left\{w \in U^{n}: \operatorname{dist}\left(w, \partial U^{n}\right) \geq \delta\right\}$, and observe that $G$ is a compact subset of $\mathbb{C}^{n}$.

Then by Lemmas 2.3, 2.4, and 2.6, and by Proposition 3.1, we deduce

$$
\begin{aligned}
\left\|C_{\phi}\right\|_{e} \leq & \sup _{\|f\|=1} \sup _{z \in G_{1}} \sum_{k, l=1}^{n}\left|\frac{\partial \phi_{l}}{\partial z_{k}}(z)\right| \frac{\left(1-\left|z_{k}\right|^{2}\right)^{q}}{\left(1-\left|\phi_{l}(z)\right|^{2}\right)^{p}}\left|\frac{\partial\left(I-K_{m}\right) f}{\partial w_{l}}(\phi(z))\right|\left(1-\left|\phi_{l}(z)\right|^{2}\right)^{q} \\
& +C \sup _{\|f\|=1} \sup _{z \in G_{2}} \sum_{l=1}^{n}\left(1-\left|\phi_{l}(z)\right|^{2}\right)^{p}\left|\frac{\partial\left(I-K_{m}\right) f}{\partial w_{l}}(\phi(z))\right| \\
& +\sup _{\|f\|=1}\left|f(\phi(0))-f\left(\frac{m-1}{m} \phi(0)\right)\right| \\
\leq & \left\|I-K_{m}\right\| \sup _{z \in G_{1}} \sum_{k, l=1}^{n}\left|\frac{\partial \phi_{l}}{\partial z_{k}}(z)\right| \frac{\left(1-\left|z_{k}\right|^{2}\right)^{q}}{\left(1-\left|\phi_{l}(z)\right|^{2}\right)^{p}} \\
& +C \sup _{\|f\|=1} \sup _{z \in G_{2}} \sum_{l=1}^{n}\left(1-\left|\phi_{l}(z)\right|^{2}\right)^{p}\left|\frac{\partial\left(I-K_{m}\right) f}{\partial w_{l}}(\phi(z))\right| \\
& +\sup _{\|f\|=1}\left|f(\phi(0))-f\left(\frac{m-1}{m} \phi(0)\right)\right|
\end{aligned}
$$




$$
\begin{aligned}
& \leq 2 \sup _{z \in G_{1}} \sum_{k, l=1}^{n}\left|\frac{\partial \phi_{l}}{\partial z_{k}}(z)\right| \frac{\left(1-\left|z_{k}\right|^{2}\right)^{q}}{\left(1-\left|\phi_{l}(z)\right|^{2}\right)^{p}} \\
& \quad+C \sup _{\|f\|=1} \sup _{z \in G_{2}} \sum_{l=1}^{n}\left(1-\left|\phi_{l}(z)\right|^{2}\right)^{p}\left|\frac{\partial\left(I-K_{m}\right) f}{\partial w_{l}}(\phi(z))\right| \\
& \quad+\sup _{\|f\|=1}\left|f(\phi(0))-f\left(\frac{m-1}{m} \phi(0)\right)\right| .
\end{aligned}
$$

Denoting the second term and third term of the right-hand side of (3.27) by $I_{1}$ and $I_{2}$, then Theorem 1.1 is proved if we can prove

$$
\lim _{m \rightarrow \infty} I_{1}=0, \quad \lim _{m \rightarrow \infty} I_{2}=0
$$

To do this, let $z \in G_{2}$ and $w=\phi(z) \in G$. Then

$$
\begin{aligned}
I_{1} \leq C & \sup _{\|f\|=1} \sup _{w \in G} \sum_{l=1}^{n}\left(1-\left|w_{l}\right|^{2}\right)^{p}\left|\frac{\partial f}{\partial w_{l}}(w)-\left(1-\frac{1}{m}\right) \frac{\partial f}{\partial w_{l}}\left(\left(1-\frac{1}{m}\right) w\right)\right| \\
\leq & C \sup _{\|f\|=1} \sup _{w \in G} \sum_{l=1}^{n}\left(1-\left|w_{l}\right|^{2}\right)^{p}\left|\frac{\partial f}{\partial w_{l}}(w)-\frac{\partial f}{\partial w_{l}}\left(\left(1-\frac{1}{m}\right) w\right)\right| \\
& +\frac{C}{m} \sup _{\|f\|=1} \sup _{w \in G} \sum_{l=1}^{n}\left(1-\left|w_{l}\right|^{2}\right)^{p}\left|\frac{\partial f}{\partial w_{l}}\left(\left(1-\frac{1}{m}\right) w\right)\right| \\
\leq & C \sup _{\|f\|=1} \sup _{w \in G} \sum_{l=1}^{n}\left(1-\left|w_{l}\right|^{2}\right)^{p}\left|\frac{\partial f}{\partial w_{l}}(w)-\frac{\partial f}{\partial w_{l}}\left(\left(1-\frac{1}{m}\right) w\right)\right|+\frac{C}{m} .
\end{aligned}
$$

Letting $w=\left(w_{1}, w_{2}, \ldots, w_{n-1}, w_{n}\right)$, for $m$ large enough, we have

$$
\begin{aligned}
& \left|\frac{\partial f}{\partial w_{l}}(w)-\frac{\partial f}{\partial w_{l}}\left(\left(1-\frac{1}{m}\right) w\right)\right| \\
& \leq \sum_{j=1}^{n} \mid \frac{\partial f}{\partial w_{l}}\left(\left(1-\frac{1}{m}\right) w_{1}, \ldots,\left(1-\frac{1}{m}\right) w_{j-1}, w_{j}, \ldots, w_{n}\right) \\
& \quad-\frac{\partial f}{\partial w_{l}}\left(\left(1-\frac{1}{m}\right) w_{1}, \ldots,\left(1-\frac{1}{m}\right) w_{j}, w_{j+1}, \ldots, w_{n}\right) \mid \\
& =\sum_{j=1}^{n}\left|\int_{(1-(1 / m)) w_{j}}^{w_{j}} \frac{\partial^{2} f}{\partial w_{l} \partial w_{j}}\left(\left(1-\frac{1}{m}\right) w_{1}, \ldots,\left(1-\frac{1}{m}\right) w_{j-1}, \zeta, w_{j+1}, \ldots, w_{n}\right) d \zeta\right| \\
& \leq \frac{1}{m} \sum_{j=1}^{n} \sup _{w \in G}\left|\frac{\partial^{2} f}{\partial w_{l} \partial w_{j}}(w)\right| .
\end{aligned}
$$

Denote $G_{3}$ by the set $\left\{w \in U^{n}: \operatorname{dist}\left(w, \partial U^{n}\right)>\delta / 2\right\}$. Then $G \subset G_{3} \subset \overline{G_{3}} \subset U^{n}$. 
Since $\operatorname{dist}\left(G, \partial G_{3}\right)=\delta / 2$, then by Lemma $2.9,(3.30)$ gives

$$
\left|\frac{\partial f}{\partial w_{l}}(w)-\frac{\partial f}{\partial w_{l}}\left(\left(1-\frac{1}{m}\right) w\right)\right| \leq \frac{2 n \sqrt{n}}{m \delta} \max _{z \in G_{3}}\left|\frac{\partial f}{\partial w_{l}}(w)\right|
$$

On the other hand, on the unit ball of $\mathscr{S} P\left(U^{n}\right)$, we have

$$
\sup _{z \in G_{3}}\left(1-\left|w_{l}\right|^{2}\right)^{p}\left|\frac{\partial f}{\partial w_{l}}(w)\right|=\sup _{\operatorname{dist}\left(w, \partial U^{n}\right)>\delta / 2}\left(1-\left|w_{l}\right|^{2}\right)^{p}\left|\frac{\partial f}{\partial w_{l}}(w)\right| \leq\|f\|_{p}=1,
$$

namely,

$$
\sup _{z \in G_{3}}\left|\frac{\partial f}{\partial w_{l}}(w)\right| \leq \frac{1}{\left(1-(\delta / 2)^{2}\right)^{p}}=\frac{4^{p}}{\left(4-\delta^{2}\right)^{p}}
$$

Combining (3.29), (3.31), and (3.33)), it follows that

$$
I_{1} \leq \frac{2 n \sqrt{n} C}{m \delta} \frac{4^{p}}{\left(4-\delta^{2}\right)^{p}}+\frac{C}{m}
$$

and $\lim _{m \rightarrow \infty} I_{1}=0$.

Now we can prove $\lim _{m \rightarrow \infty} I_{2}=0$. In fact,

$$
f(\phi(0))-f\left(\frac{m-1}{m} \phi(0)\right)=\int_{(m-1) / m}^{1} \frac{d f(t \phi(0))}{d t} d t=\sum_{l=1}^{n} \int_{(m-1) / m}^{1} \phi_{l}(0) \frac{\partial f}{\partial \zeta_{l}}(t \phi(0)) d t .
$$

By Lemma 2.1, it follows that for any compact subset $K \subset U^{n},|f(z)| \leq C_{K}\|f\|_{p}=C_{K}$. Let $K=\left\{z \in U^{n}:\left|z_{i}\right| \leq\left|\phi_{i}(0)\right|, i=1, \ldots, n\right\}$, So

$$
\left|f(\phi(0))-f\left(\frac{m-1}{m} \phi(0)\right)\right| \leq \sum_{l=1}^{n}\left|\phi_{l}(0)\right| \int_{(m-1) / m}^{1} C_{K} d t \leq n C_{K}\left(1-\frac{m-1}{m}\right)=\frac{n C_{K}}{m}
$$

so $I_{2} \leq n C_{K} / m \rightarrow 0$. Thus letting first $m \rightarrow \infty$ and then $\delta \rightarrow 0$ in (3.27), we get the upper estimate of $\left\|C_{\phi}\right\|_{e}$ :

$$
\left\|C_{\phi}\right\|_{e} \leq 2 \lim _{\delta \rightarrow 0} \sup _{\operatorname{dist}\left(\phi(z), \partial U^{n}\right)<\delta} \sum_{k, l=1}^{n}\left|\frac{\partial \phi_{l}}{\partial z_{k}}(z)\right| \frac{\left(1-\left|z_{k}\right|^{2}\right)^{q}}{\left(1-\left|\phi_{l}(z)\right|^{2}\right)^{p}} .
$$

Now the proof of Theorem 1.1 is finished. 


\section{Some corollaries}

The following three corollaries follow from Theorem 1.2.

Corollary 4.1. Let $\phi=\left(\phi_{1}, \ldots, \phi_{n}\right)$ be a holomorphic self-map of $U^{n}$. Then $C_{\phi}$ : $\mathscr{B}^{p}\left(U^{n}\right)\left(\mathscr{B}_{0}^{p}\left(U^{n}\right)\right.$ or $\left.\mathscr{B}_{0 *}^{p}\left(U^{n}\right)\right) \rightarrow \mathscr{B}^{q}\left(U^{n}\right)$ is compact if and only if

$$
\sum_{k, l=1}^{n}\left|\frac{\partial \phi_{l}}{\partial z_{k}}(z)\right| \frac{\left(1-\left|z_{k}\right|^{2}\right)^{q}}{\left(1-\left|\phi_{l}(z)\right|^{2}\right)^{p}} \leq C
$$

for all $z \in U^{n}$ and (1.12) holds.

Proof. By Lemma 2.3, we know $C_{\phi}: \mathscr{B}^{p}\left(U^{n}\right)\left(\mathscr{B}_{0}^{p}\left(U^{n}\right)\right.$ or $\left.\mathscr{B}_{0 *}^{p}\left(U^{n}\right)\right) \rightarrow \mathscr{B}^{q}\left(U^{n}\right)$ is bounded. It follows from Theorem 1.2 that $C_{\phi}: \mathscr{B}^{p}\left(U^{n}\right)\left(\mathscr{B}_{0}^{p}\left(U^{n}\right)\right.$ or $\left.\mathscr{B}_{0 *}^{p}\left(U^{n}\right)\right) \rightarrow \mathscr{B}^{q}\left(U^{n}\right)$ is compact.

Conversely, if $C_{\phi}: \mathscr{B}^{p}\left(U^{n}\right)\left(\mathscr{B}_{0}^{p}\left(U^{n}\right)\right.$ or $\left.\mathscr{R}_{0 *}^{p}\left(U^{n}\right)\right) \rightarrow \mathscr{B}^{q}\left(U^{n}\right)$ is compact, it is clear that $C_{\phi}: \mathscr{B}^{p}\left(U^{n}\right)\left(\mathscr{S}_{0}^{p}\left(U^{n}\right)\right.$ or $\left.\mathscr{B}_{0 *}^{p}\left(U^{n}\right)\right) \rightarrow \mathscr{B}^{q}\left(U^{n}\right)$ is bounded, by Theorem 1.2, (1.12) holds.

Corollary 4.2. Let $\phi=\left(\phi_{1}, \ldots, \phi_{n}\right)$ be a holomorphic self-map of $U^{n}$. Then $C_{\phi}$ : $\mathscr{B}_{0 *}^{p}\left(U^{n}\right)\left(\mathscr{B}_{0}^{p}\left(U^{n}\right)\right) \rightarrow \mathscr{B}_{0 *}^{q}\left(U^{n}\right)$ is compact if and only if $\phi_{l} \in \mathscr{B}_{0 *}^{q}\left(U^{n}\right)$ for every $l=1$, $2, \ldots, n$ and (1.12) holds.

The proof follows from Lemma 2.4 .

Corollary 4.3. Let $\phi=\left(\phi_{1}, \ldots, \phi_{n}\right)$ be a holomorphic self-map of $U^{n}$. Then $C_{\phi}: \mathscr{B}_{0}^{p}\left(U^{n}\right) \rightarrow$ $\mathscr{B}_{0}^{q}\left(U^{n}\right)$ is compact if and only if $\phi_{l} \in \mathscr{B}_{0}^{q}\left(U^{n}\right)$ for every $l=1,2, \ldots, n$ and (1.12) holds.

The proof follows from Lemma 2.6.

\section{Acknowledgments}

The authors would like to thank the editor and referee(s) for helpful comments on the manuscript. The first author is supported in part by the National Natural Science Foundation of China (Grants no. 10671141 and no. 10371091) and LiuHui Center for Applied Mathematics, Nankai University \& Tianjin University.

\section{References}

[1] K. Madigan and A. Matheson, Compact composition operators on the Bloch space, Transactions of the American Mathematical Society 347 (1995), no. 7, 2679-2687.

[2] A. Montes-Rodríguez, The essential norm of a composition operator on Bloch spaces, Pacific Journal of Mathematics 188 (1999), no. 2, 339-351.

[3] J. H. Shapiro, The essential norm of a composition operator, Annals of Mathematics 125 (1987), no. 2, 375-404.

[4] J. H. Shi and L. Luo, Composition operators on the Bloch space of several complex variables, Acta Mathematica Sinica. English Series 16 (2000), no. 1, 85-98.

[5] R. M. Timoney, Bloch functions in several complex variables. I, The Bulletin of the London Mathematical Society 12 (1980), no. 4, 241-267. 


\section{Essential norm of composition operators}

[6] B Bloch functions in several complex variables. II, Journal für die reine und angewandte Mathematik 319 (1980), 1-22.

[7] Z. Zhou, Composition operators on the Lipschitz space in polydiscs, Science in China. Series A 46 (2003), no. 1, 33-38.

[8] Z. Zhou and J. H. Shi, Compact composition operators on the Bloch space in polydiscs, Science in China. Series A 44 (2001), no. 3, 286-291.

[9] Composition operators on the Bloch space in polydiscs, Complex Variables 46 (2001), no. $1,73-88$.

[10] Compactness of composition operators on the Bloch space in classical bounded symmetric domains, The Michigan Mathematical Journal 50 (2002), no. 2, 381-405.

[11] _ The essential norm of a composition operator on the Bloch space in polydiscs, Chinese Annals of Mathematics. Series A 24 (2003), no. 2, 199-208, Chinese Journal of Contemporary Mathematics 24 (2003), no. 2, 175-186.

[12] Z. Zhou and H. G. Zeng, Composition operators between p-Bloch space and q-Bloch space in the unit ball, Progress in Natural Science 13 (2003), no. 3, 233-236.

[13] K. Zhu, Spaces of Holomorphic Functions in the Unit Ball, Graduate Texts in Mathematics, vol. 226, Springer, New York, 2005.

Zehua Zhou: Department of Mathematics, Tianjin University, Tianjin 300072, China

E-mail address: zehuazhou2003@yahoo.com.cn

Yan Liu: Department of Mathematics, Tianjin University, Tianjin 300072, China

E-mail address: maple.ly@163.com 